\title{
Rotationally induced transitions in small clusters
}

\author{
Ersin Yurtsever* \\ Chemistry Department, Koç University, Rumelifeneri Yolu, Sariyer, Istanbul 80900, Turkey \\ (Received 22 February 2000; revised manuscript received 6 September 2000; published 18 December 2000)

\begin{abstract}
The dynamics of an $\mathrm{Ar}_{6}$ cluster held together by Lennard-Jones forces is studied classically. The development of chaotic dynamics is mainly followed by a calculation of the maximum Lyapunov exponent (MLE). Initial momentum vectors are chosen from the eigenvectors of the Hessian of the potential energy so that rotating and nonrotating clusters can be studied systematically. It is found that the dependence of MLE on the total energy is considerably different for rotational and vibrational excitations. As the magnitude of the angular momentum increases, sharp transitions in MLE are observed. These transitions are explained in terms of the changes of the topology of the effective rovibrational potential energy surface and the dynamic equilibration between the global and local minima.
\end{abstract}

DOI: 10.1103/PhysRevE.63.016202

PACS number(s): 05.45.Pq, 05.70.Fh, 36.40.-c

\section{INTRODUCTION}

The dynamics of small clusters offers a powerful computational laboratory tool for understanding several interesting phenomena, such as isomerizations, phase transitions, and phase coexistence [1-3]. Due to the relatively small number of degrees of freedom, a detailed analysis of their dynamics is feasible, at least in classical mechanical approaches. Unlike bulk materials, the local environment of individual atoms shows a great deal of inhomogeneities which results in very rich dynamic behavior. One of the basic reasons behind this inhomogeneity is the presence of a large number of local minima and saddle points of the potential energy surfaces (PES) with distinct geometrical structures. For a clear understanding of the dynamics along the PES, one needs to know the connectivity between such minima and saddle points as well as the topology of the surfaces close to them. Once the number of optimum structures reaches a certain level, statistical interpretations of the PES topology become necessary in order to elucidate the kinetics [4].

In all these studies it has been observed that if sufficient kinetic energy is stored in a cluster, its classical dynamics shows chaotic behavior [5,6]. The extent of the irregularity can be measured in terms of the Lyapunov exponent spectrum or its components, such as the maximum Lyapunov exponent $(\lambda)$ or Kolmogorov $(\mathrm{K})$ entropy, which is the sum of all positive exponents. The variation of such measures as functions of the energy (or average temperature) usually points to qualitative changes in the dynamics of the system. The earliest work on Lyapunov exponents of Lennard-Jones (LJ) systems belongs to Posch and Hoover [7,8], where the Lyapunov spectra of dense fluids have been studied under different conditions. On the other hand, the majority of the results on small, isolated clusters have been reported by Berry and co-workers. In early studies, the Ar trimer has been analyzed extensively and it has been found that the maximum Lyapunov exponent (MLE) increases with energy as in countless other Hamiltonian systems. Once the total energy is around $-2 / 3 \epsilon$, where $\epsilon$ is the well depth of the $\mathrm{LJ}$

*Email address: eyurtsev@ku.edu.tr potential function, a drop in MLE is observed, which is explained by the accessibility of the relatively flat saddle point of the trimer corresponding to linear structure $[5,9]$. We also observed this unusual drop in our studies of $\mathrm{Ar}_{3}$. Additionally, we have reported that three-body terms in the potential energy stabilize and reduce the MLE [10]. Berry has reported a similar observation for $\mathrm{LJ}_{4}$ where a flat saddle regularizes the dynamics. However, when the cluster size is increased, the saddle points usually become sharper and no additional regularity is introduced into the system [9]. Obviously, since the cluster spends so little time around these sharp saddle points, the time averages of trajectories are hardly affected by their presence.

In studies of small clusters, it is especially important to understand the relationship between the Lyapunov exponents and the phase transitions. In a detailed description of the melting process, it is explained that even for small clusters a liquidlike behavior is observed if there are stable isomers other than the global minimum [11]. The passage of the cluster between these global and local minima produces a less rigid structure where the time scales of the vibrational motion and switching between local isomers are distinguishable. Calculations on larger clusters up to $\mathrm{Ar}_{14}$ also show that the topography of the potential surface and the melting-freezing processes are closely related [12]. Again, the number of near-degenerate minima and saddle points and the rearrangement mechanisms connecting these optimum points play a crucial role in the phase transitions. Since MLE or $K$ entropy can be used in the characterization of the qualitative changes of the dynamics, it is important to understand the behavior of these measures in the phase transition and/or coexistence regime. Nayak et al. have reported a dramatic increase in MLE of $\mathrm{LJ}_{n}$ with respect to temperature in the phase transition regime for $n=7,13,55$ [13]. Their explanation for this abrupt change is the sudden increase in the available phase-space volume. They claim that the slope of the MLE with respect to the energy is a more sensitive measure for the phase transitions than the MLE itself [14]. In bulk systems, the MLE and the energy show a jump at the same temperature, implying first-order transitions [15].

On the other hand, extensive studies by Calvo and Labastie on LJ systems as well as ionic clusters fail to produce 
such sharp changes in MLE upon melting [16,17]. The variation of MLE almost linearly increases with energy for LJ clusters smaller than $n=38$. The only detectable change in the slope of MLE is the onset of the first isomerization for ionic clusters. Our simulations on LJ clusters on $n \leqslant 20$ also did not show any significant change in MLE or its derivative around the phase transition regime.

The initial conditions may be very important for the calculation of the MLE and characterization of the dynamics. We have reported a set of calculations on $\mathrm{LJ}_{3}$ where either the energy is stored randomly in bonds with $L=0$ or ordered rotations are imposed on the cluster with $L>0[18,19]$. Our conclusion was that the initial partitioning of the kinetic energy in vibrational and rotational motion determines the general characterization of the dynamics. Specifically, the more energy stored in the vibrational motion, the more chaotic the trajectories become.

In this work, we present our extensive results on rotating and nonrotating $\mathrm{LJ}_{6}$. It is our aim to characterize the dynamics by partitioning the phase space specifically in terms of angular momentum. For this purpose, the geometry of the cluster, the direction, and the magnitude of the atomic momentum vectors are used to identify qualitatively different regions of the phase space. Through specifically chosen initial momentum vectors, it is possible to generate conditions where the cluster is given an initial rotational kick or a specific normal mode of the cluster is excited. In the case of the former, the energy is redistributed in time due to various coupling mechanisms between the rotational and vibrational motions. Here we present the variation of the MLE as a function of the angular momentum and the total energy in order to study the relationships between these measures and the geometrical changes. In our earlier studies of $\mathrm{LJ}_{3}$ we have shown that the initial partitioning of the kinetic energy between the vibrational and rotational motions was the dominant factor determining the extent of the chaotic behavior $[10,18,19]$. That is, even though the angular velocity, the inertia tensor, and the angular kinetic energy change continuously as functions of time [20], the energy in the vibrational motion (at $t=0$ only the kinetic energy is nonzero, since the clusters rest at the minimum) defines the extent of the irregularity. If a sufficient amount of energy cannot be transferred to the vibrational motion, the dynamics remains regular.

The phase space of $\mathrm{Ar}_{6}$ is comparatively more difficult to map. However, we resolve that difficulty by systematically changing the total energy for each rotational and vibrational excitation. In the case of the rotating clusters, there exists an additional complexity due to the changes of the potential energy surface by the centrifugal forces. A detailed discussion of these changes in terms of the effective rovibrational potential energy is given by $\mathrm{Li}$ and Jellinek [21]. They have observed that centrifugal distortion due to nonzero angular momentum may significantly change the topology of the potential surface and can even result in isomerizations or fragmentations. Similarly the effects of such forces on the equilibrium geometry, rearrangements of the cluster, spectroscopic constants, and rate constants are presented by Miller and Wales [22]. One of the important findings of their study was that nonrigidity of the clusters can lead to the instability of rotations. The corrections to the spectroscopic constants were also discussed by Lohr and Huben [23], who studied rotating clusters in detail, including the heterogeneous trimers and tetramers [24] and noted the changes of the configurational space for rotating systems. In order to detect the changes in the dynamics for rotating clusters, we proceed to define the effective rovibrational surface as in $[21,22]$. If a cluster is rotating around its center of mass, its angular momentum vector is conserved in time; however, the moment of inertia and the angular velocity are timedependent observables.

$$
\vec{L}=\vec{\omega}(t) I(t),
$$

where $\vec{\omega}$ is the angular velocity and $I$ is the moment of the inertia tensor. Since the angular velocity can be written as

$$
\vec{\omega}(t)=I(t)^{-1} \vec{L},
$$

we can write the rotational kinetic energy as

$$
E^{r o t}=\frac{1}{2} \vec{L} I^{-1} \vec{L}
$$

Since the moment of the inertia tensor $(I)$ is a function of coordinates only, the effective rovibrational potential energy function can be defined as

$$
V_{e f f}=V_{L J}+\frac{1}{2} \vec{L} I^{-1} \vec{L}
$$

where $V_{L J}$ is the potential energy of the nonrotating LJ cluster. The trajectory lies on this effective rovibrational potential surface and the number of optimum points as well as their characterization can be significantly different than the nonrotating case.

In order to find the optimum points of the rovibrational potential energy surface, we have used the program OPTIM, which was kindly provided by D. J. Wales. Subroutines to calculate the analytical gradient and the Hessian are added. The gradient in Cartesian coordinates is given as

$$
\frac{\partial}{\partial q_{k}} E^{r o t}=-\frac{1}{2} \vec{L}\left(I^{-1} \frac{\partial I}{\partial q_{k}} I^{-1}\right) \vec{L} .
$$

The Hessian can then can be written as

$$
\begin{aligned}
\frac{\partial^{2}}{\partial q_{l} \partial q_{k}} E^{r o t}= & \frac{1}{2} \vec{L}\left(I^{-1} \frac{\partial I}{\partial q_{l}} I^{-1} \frac{\partial I}{\partial q_{k}} I^{-1}-I^{-1} \frac{\partial^{2} I}{\partial q_{l} \partial q_{k}} I^{-1}\right. \\
& \left.+I^{-1} \frac{\partial I}{\partial q_{k}} I^{-1} \frac{\partial I}{\partial q_{l}} I^{-1}\right) \vec{L} .
\end{aligned}
$$

The number of optimum points as well as their energy depend strongly on the magnitude of the angular momentum vector $\vec{L}$ and, in a more subtle way, on its direction. In general, the energy differences between the minima of $V_{L J}$ and the effective potential are proportional to $L^{2}$ for small $L$ values. 
For rotating clusters, variations in MLE can be studied as functions of the total energy of the cluster, angular momentum $L$, or the energy relative to the global minimum for the specific $L$. These three parameters are closely related as

$$
E^{T o t}-V_{0}=\Delta V+\frac{1}{2} \sum p_{i}^{2},
$$

where $V_{0}$ is the potential energy of the global minimum and $\Delta V$ is the potential energy difference between the minimum geometry (function of $L$ ) and the starting geometry. Initially $\vec{p}$ is proportional to a rotational eigenvector: $\vec{L}=\vec{r} \times \vec{p}$; therefore, at $t=0$, we can write that

$$
E^{T o t}-V_{0}=\Delta V+\frac{1}{2} \vec{L} I^{-1} \vec{L}
$$

For small $L, \Delta V$ is very small and $E^{T o t}-V_{0}$ is proportional to the square of the angular momentum. As $L$ increases, $V_{0}$ changes; however, $E^{T o t}-V_{0}$ still remains roughly proportional to $L^{2}$. Consequently, the plots of MLE versus any of these three functions give the same information, apart from a quadratic transformation.

\section{CALCULATiONS}

The dynamics of clusters is followed by the integration of the Hamilton's equations of motion in time. The four-step Runge-Kutta integration is used to obtain the time evolution of coordinates and momenta. Even though predictorcorrector-type integrators are more cost efficient, the use of Runge-Kutta accurately guarantees the conservation of energy and momentum at the initial stages. The scaled version of the LJ potential is used $(\epsilon=1, \sigma=1)$; therefore, the reported energy values are in terms of $\epsilon$ and time, and angular momentum is in generalized units (gu). The time step is chosen to be $0.0025 \mathrm{gu}$ and the equations of motion are integrated for $10^{6}$ steps. The Lyapunov exponents are calculated by the tangent space method $[5,25]$. When the differential equations driving the dynamics are known, the complete Lyapunov spectrum can be calculated within the tangent space. An infinitesimal $6 \mathrm{~N}$-dimensional hyperspheroid is allowed to evolve along with the cluster. The equations of motion for the axes of the hyperspheroid can be cast into linearized forms, since the distances between the initial point and the surface of the hyperspheroid in the phase space are very small at the beginning but diverge exponentially. By tracking the lengths of the axes of the hyperspheroid, the essential dynamics can be captured. Since the equations for the axes are in linear form, it is not necessary to work with infinitesimal lengths and any arbitrary (preferably orthogonal) matrix can be used to define the initial conditions. These axes in essence represent close-lying trajectories and they have to be orthonormalized periodically to prevent overflows and underflows. In this work, Gram-Schmidt orthonormalizations are applied at every 200 steps. MLE is very much independent of the frequency of the orthonormalization. During the integration, the conservation of energy is on the order of $10^{-7} \epsilon$. For Hamiltonian systems, the sum of all exponents must be zero, since the phase-space volume is conserved. For the work reported here the sum of exponents is less than $10^{-8}$ bits/time (gu) when the complete spectrum is calculated.

The tangent-space method, besides being a relatively overflow-free method, allows easy access to the MLE without calculating the full Lyapunov exponent spectrum. Within this methodology, it is possible to start with a single random axis which is usually a normalized eigenvector of length $6 \mathrm{~N}$, instead of fully defining the initial conditions for the hyperspheroid; this eigenvector eventually collapses into one corresponding to the maximum stretching, hence the MLE. This conjecture has been shown to be correct for Brownian motion [26] and was observed to be valid in our simulations. When the dynamics is chaotic with large Lyapunov exponents, MLE can be calculated by solving the linear equations for a single trajectory (axis). Only for the regular regime, when MLE is nearly zero, calculation of the exact MLE requires not one but usually $2-3$ axes. However, this problem can be solved with longer integration times.

In this work we present results on $\mathrm{Ar}_{6}$ simulations. The PES of nonrotating $\mathrm{Ar}_{6}$ has two minima and three first-order saddle points with energies of $-12.71206,-12.30293$, $-12.07903,-11.63031$, and $-11.33403 \epsilon$. The initial conditions are chosen such that the geometry of the cluster corresponds to one of these optimal points. In order to span the phase space systematically and analyze the effects of the rotational motion, the following procedure has been applied. The Hessian of the Lennard-Jones potential at the starting geometry is diagonalized to generate $3 N-6$ eigenvectors for normal modes as well as three vectors for the translational and three for the rotational motion. The eigenvectors corresponding to the translational motion are identified as those with zero frequency and nonzero linear momentum. These modes are excluded from simulations. Three eigenvectors with zero frequency and nonzero angular momentum describe the rigid-body rotations of the chosen geometry.

Initial conditions for the sample set of trajectories describe two distinct types of motion. One of these types mimicks the photoexcitation of a normal mode. This is achieved by choosing one of the normal-mode eigenvectors as the initial momentum vector. In this manner the energy is stored in a single specific mode and all other modes are initially inactive. Similarly, when the eigenvectors are chosen from one of the three rotational modes, the initial conditions describe a rotational kick given to a nonvibrating cluster. In these cases, as the system is nonrigid, the vibrational motion sets in very quickly. We denote two types of initial conditions: vibrational and rotational excitations, respectively. For both excitation types, the energy flows between the kinetic and potential energy. Additionally, there may be a strong energy transfer between rotational and vibrational kinetic energy for the rotational excitations. The strength of the unimolecular energy redistribution within various types of motion and modes defines the irregularity of the dynamics.

Once the coordinates and momenta are selected, the kinetic energy is scaled to cover a wide range of energy. The simulations are carried out in the energy range of $-12.0 \epsilon$ $\leqslant E \leqslant-7.5 \epsilon$. Within this energy regime, it is possible for 


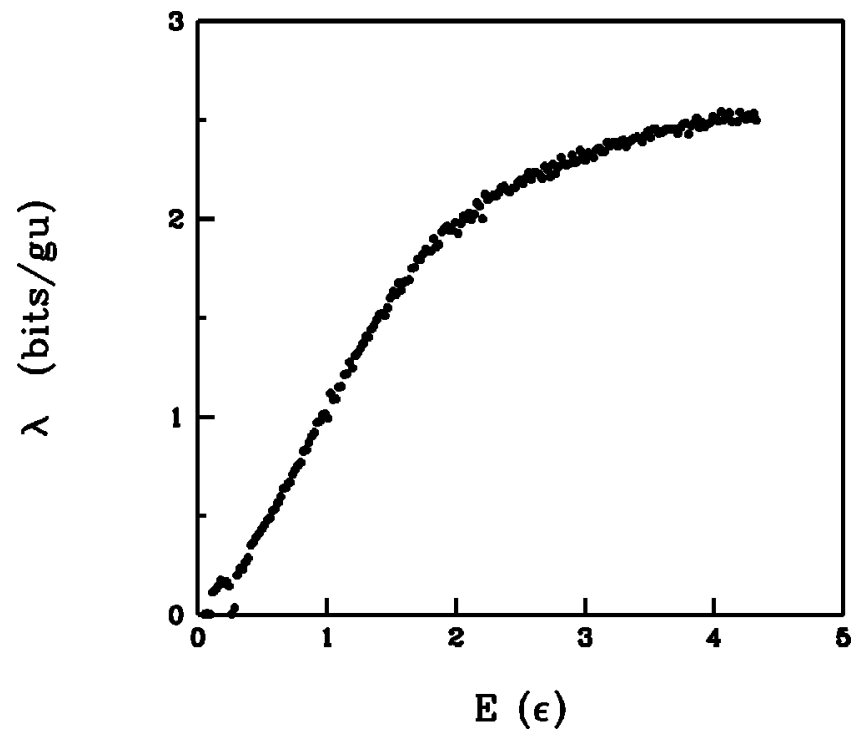

FIG. 1. MLE as a function of the total energy for the excitations of the lowest-frequency normal mode.

some trajectories to result in evaporation, and when this happens the trajectory is discarded from the set.

\section{MAXIMUM LYAPUNOV EXPONENTS}

In Fig. 1 the MLE versus total energy (relative to the energy of the cluster at rest) plot for the smallest frequency vibrational normal mode is given for trajectories starting from the global minimum of $\mathrm{Ar}_{6}$. The dependence of MLE on energy for other vibrational excitations as well as those starting from local minima are very similar, especially at the high-energy regime. The MLE increases smoothly with increasing energy. A study of the convergence of trajectories in this region reveals that there exists a very low energy threshold below which the normal-mode description is accurately preserved.

On the other hand, rotational excitations in $\mathrm{Ar}_{6}$ show quite different characteristics. Out of three rotational modes obtained from the Hessian, two are distinct. One of them corresponds to a rotation around the $C_{4}$ axis and the other two are linear combinations of rotations around two $C_{2}$ axes. Since the eigenvalues corresponding to these axes are zero, we obtain a random mixture of these two $C_{2}$ as eigenvectors; however, the general characteristics of the dynamics do not change significantly.

In Figs. 2(a) and 2(b), MLE for rotational excitations starting from the global minimum of the nonrotating cluster are plotted for both rotation types as functions of the magnitude of $\vec{L}$. Sharp "transitions" around $|L|=4.5$ gu are observed in these figures. In order to understand the dynamics, one needs to look at the partitioning of the total energy. Following the notation of $\mathrm{Li}$ and Jellinek [21], $E^{T o t}=V$ $+E^{T h}+E^{R o t}$, where $E^{T h}$ is the remaining kinetic energy stored in vibrational motion. From the initial conditions, it seems that all the kinetic energy is in the rotational motion and the thermal (vibrational) energy is zero. However, the cluster does not start at the minimum geometry of the rovi-
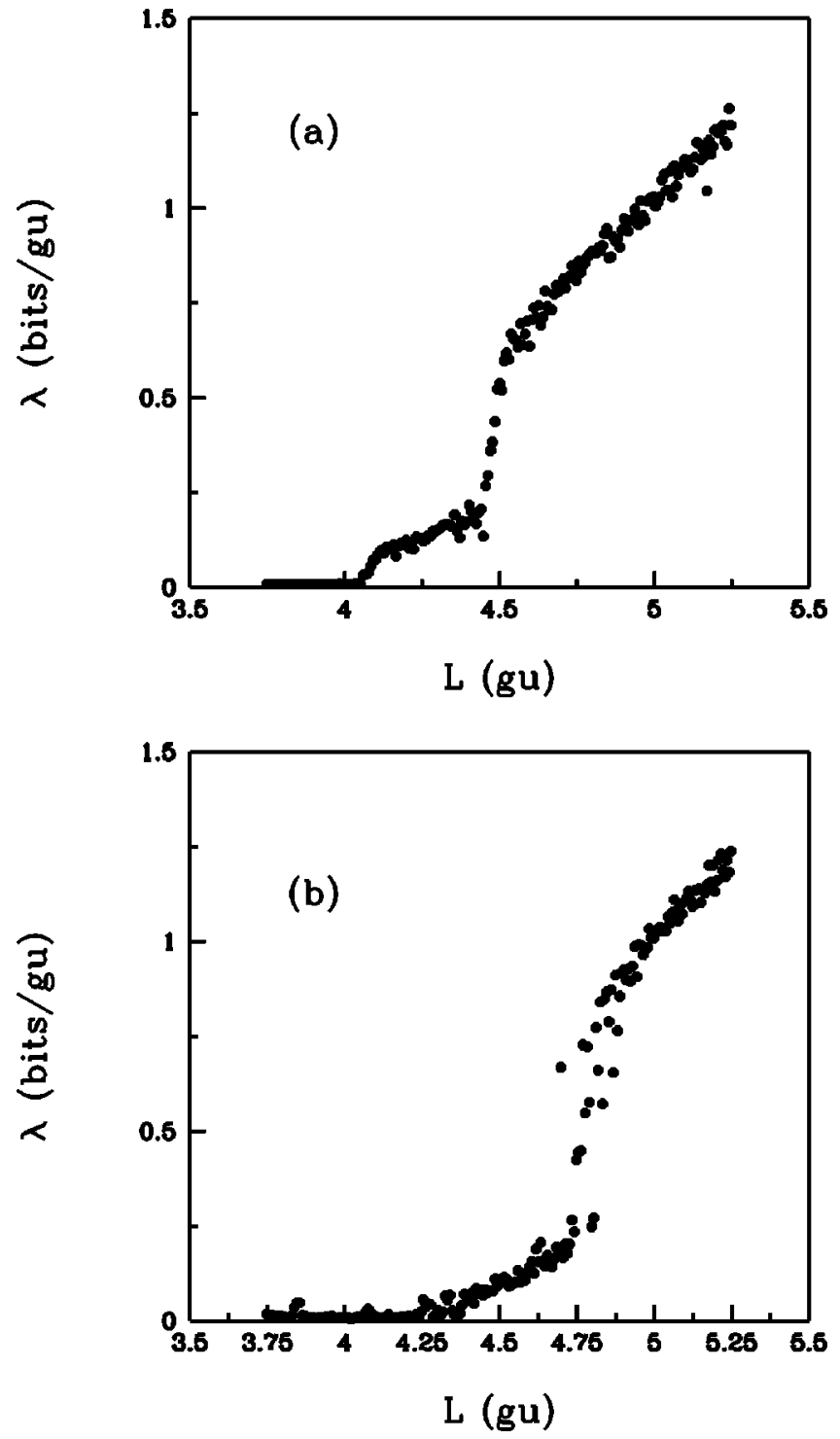

FIG. 2. MLE dependence on angular momentum. (a) Rotation around $C_{4}$. (b) Rotation around $C_{2}$.

brational surface due to slight changes in the global minimum with angular momentum. Hence, $\Delta V$ is nonzero and as the trajectory moves along the $L$-dependent "rotationally adiabatic potential surface," the energy flows between $E^{T h}, E^{R o t}$, and $V$. The dynamics is characterized by the magnitude of the energy transferred between these terms. In fact, trajectories starting from the global minimum of the rovibrational surface are regular with zero MLE, since the vibrational kinetic energy remains zero.

The topology of the adiabatic potential-energy surface can be studied through several approaches. Li and Jellinek do a special thermal quenching where the angular momentum is preserved so that the cluster collapses into the equilibrium structures of the rotating system. We have utilized the eigenvector following an approach with the potential function being defined as in $V_{e f f}$. The gradient and Hessian in Cartesian coordinates are calculated analytically and the minimum structures of the rotating clusters for $L$ changing between 0 and 7 (in generalized units) are obtained. The minima and 


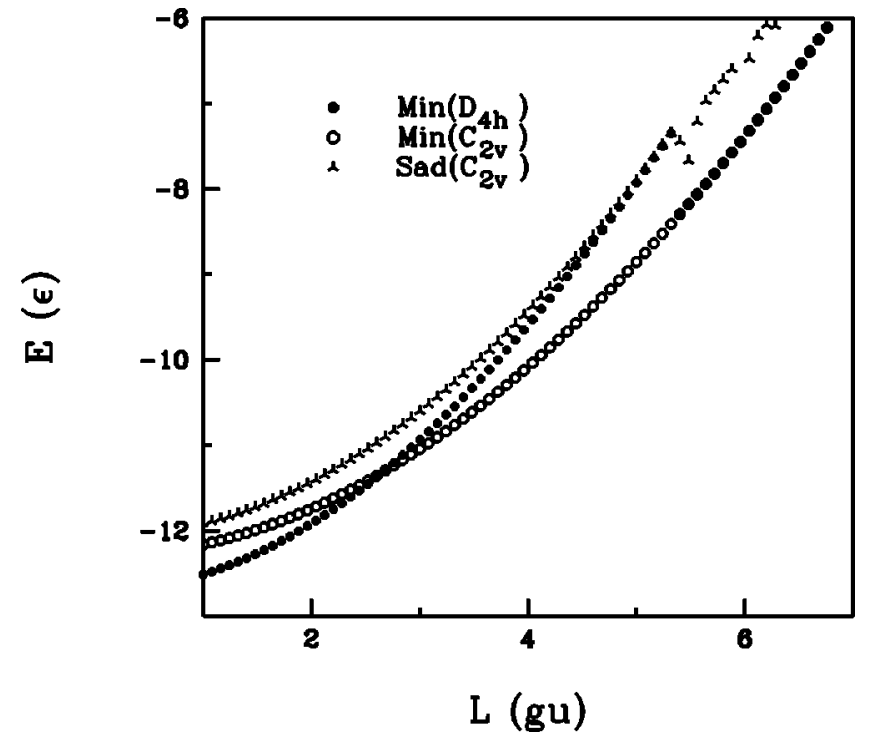

FIG. 3. Energy of the global, local minimum and the lowestenergy first-order saddle point of the $V_{\text {eff }}$ as a function of the angular momentum (rotation around $C_{4}$ ).

first-order saddle points of the $V_{L J}$ are used as initial structures for the optimization. The results of the optimization for the rotation around the $C_{4}$ axis are given in Fig. 3. The lowest three optimum points of the nonrotating cluster belong to the $O_{h}, C_{2 v}$, and $C_{2 v}$ symmetry groups. For the low-angular-momentum regime, the global minimum of the rotating cluster switches to $D_{4 h}$ symmetry (square bipyramid); the local minimum and the lowest first-order saddle point remain in the $C_{2 v}$ group. Around $L=2.5$ (corresponding to $E=-11.5 \epsilon$ ) the energy ordering of these two minima switches. This change in the global minimum of the potential energy is not really reflected in the dynamics due to the still-high-energy barrier between the global and local minima. However, as the angular momentum increases, a second feature is observed where the energy difference between the saddle point and the local minimum decreases and becomes almost degenerate around $L=4.5(E=-8.5 \epsilon)$. This change in the topology of the effective potential-energy surface is more responsible for the sharp transition observed in MLE. Trajectories starting at the $D_{4 h}$ structure (now a local minimum of the effective potential) are able to switch to the global minimum, since the energy barrier to the saddle point is very low. Thus, the sharp increase in the chaotic behavior can be accounted for in terms of the differences between the potential-energy surfaces of the rotating and nonrotating clusters.

For a more detailed analysis of the dynamics, several trajectories in the transition regime for $C_{4}$ rotation are selected and various properties are calculated. The time-series analysis of individual coordinate and moment components such as autocorrelation functions or power spectra show that the motion is definitely far from normal-mode description and not regular; however, they do not describe the mechanism. To observe the structural changes and correlate with the transition in MLE, we have carried out a "pseudoquenching.", Jellinek and Wales have proposed methods for quenching

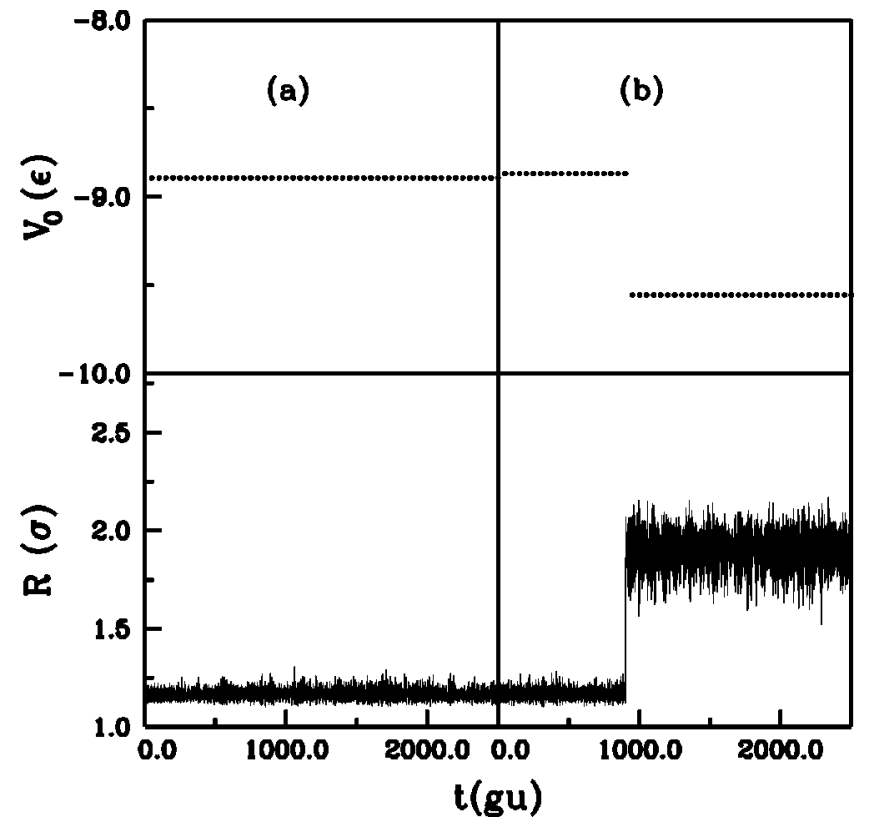

FIG. 4. Pseudoquenched energy values and the bond distance $\left(R_{12}\right)$ for two trajectories. (a) $L=4.440 \mathrm{gu}, E=-8.766 \epsilon$; (b) $L$ $=4.455 \mathrm{gu}, E=-8.739 \epsilon$.

along the rovibrational surface $[21,22]$ where the vibrational kinetic energy is extracted from clusters while keeping the angular momentum constant. In our pseudo scheme, we sample trajectories at fixed intervals to generate a set of initial structures. These structures are subjected to optimization over the $L$-dependent rotationally adiabatic surface. This procedure, though it may not be very efficient for finding all isomers, is a reasonably accurate method for determining the major structural changes during the simulation. Besides this optimization scheme, we also analyzed the bond length variations in order to extract additional evidence for the isomerization, and results from two trajectories are given in Fig. 4.

The angular momentum for the first trajectory is $L$ $=4.440 \mathrm{gu}$, which corresponds to $E=-8.766 \epsilon$ and the second one has $L=4.455 \mathrm{gu}$ and $E=-8.739 \epsilon$. They are in the transition region between regular and chaotic motion with corresponding maximum Lyapunov exponents of 0.24 and $0.55 \mathrm{bits} / \mathrm{gu}$, respectively. The initial structures obtained by sampling the first trajectory are all optimized to square bipyramidal form with an energy of $V_{0}=-8.8944 \epsilon$. The bond length shown in this figure $\left(R_{12}\right)$ corresponds to two atoms forming one side of the square. The bond length fluctuates around the mean in a quasiperiodic manner. All the other bond lengths display similar variations. The second trajectory with larger MLE has all the signatures of the isomerization. With the coordinates sampled from the first one-third of the simulation, the pseudoquenching procedure finds the square bipyramid as the minimum structure with energy $V_{0}$ $=-8.8693 \epsilon$. For the remaining part of the trajectory, the optimization locates the global minimum with $C_{2 v}$ symmetry at $E=-9.5555 \epsilon$. The bond lengths show the appropriate change in the geometry. At the beginning, the average value of $R_{12}$ is around $1.17 \sigma$, which corresponds to the bond 
length in the minimum-energy structure of the nonrotating cluster. Around $t=900 \mathrm{gu}$, the average bond length jumps to $1.9 \sigma$ and remains there. The global minimum structure obtained from optimization shows that the atoms forming the square are distorted with the rotation such that they form a trapezoid where one side is elongated (with $C_{2 v}$ symmetry) and the atoms above and below the plane move closer to the trapezoid. The bond length variations and optimum structures shown in Fig. 4 correspond to exactly the $D_{4 h}$-to- $C_{2 v}$ change and occur at the same time. The results from high $L$ and high $E$ trajectories are generally similar, showing the structural transition, except that the time for finding the global minimum is reduced as the energy is increased. At very high energy, we have observed several such transitions within the same trajectory describing a dynamic equilibrium between isomers.

We have not carried out an exhaustive search to find the actual number of distinct minima. As expected, the optimum points strongly depend on the orientation of the angular momentum vector. When the angular momentum vector coincides with one of the symmetry rotations of the cluster as it is presented here, the number of local minima does not change very drastically. However, when the angular motion destroys the symmetry, the number of local minima increases.

Calculations of clusters of up to 20 atoms display similar behavior for high-angular-momentum cases. However, the rise in the MLE is most pronounced for small sizes and as the cluster gets larger, the transitions become more gradual.

\section{CONCLUSION}

The maximum Lyapunov exponent of $\mathrm{Ar}_{6}$ is studied for initial conditions corresponding to rotating and nonrotating clusters. It is known that in the case of the nonrotating clusters, MLE is a monotonically increasing function of the total energy for larger than triatomic clusters. When the rotational motion is introduced, the dynamics changes its character and we observe sharp transitions in MLE versus angular momentum plots. The explanation lies in the changes in the topology of the potential-energy surface of rotating clusters. The effective potential now includes a new term, $\frac{1}{2} \vec{L} I^{-1} \vec{L}$, due to the centrifugal forces and consequently the number of optimum points and their energy differences are strongly influenced by the magnitude and direction of the angular momentum. In $\mathrm{Ar}_{6}$, upon rotating around the $C_{4}$ axis, the global and local minima cross around $L=2.5 \mathrm{gu}$, and the lowest saddle point becomes energetically degenerate with the local minima around $L=4.5 \mathrm{gu}$. If the cluster starting at $O_{h}$ symmetry does not have enough energy to go over the saddle point and find the global minimum, the trajectory remains regular, since there is very little energy in the vibrational motion. Those clusters which isomerize to the global minimum have relatively large vibrational energy and the dynamics becomes chaotic. We conclude by stating that sharp transitions in MLE for finite size systems can occur only due to qualitative changes in the topology of the potential-energy surface.

For higher energy (or angular momentum) cases, the cluster may visit more than one minimum or switch between two isomers and result in larger MLE. On the other hand, the simulations starting from the global minimum of the effective potential remain regular with zero Lyapunov exponents. The number of optimum points is affected by the magnitude and direction of the angular momentum vector. Symmetrybreaking rotations in particular induce an increase in the number of local minima. Larger clusters also display similar but more gradual transitions.

\section{ACKNOWLEDGMENT}

We would like to thank D. J. Wales, who kindly provided a copy of his program OPTIM for the calculation of the optimum points of the rovibrational potential energy surface.
[1] R.S. Berry, Chem. Rev. 93, 2379 (1993).

[2] R.S. Berry, J. Phys. Chem. 98, 6910 (1994).

[3] D.J. Wales, Science 271, 925 (1996).

[4] R.E. Kunz and R.S. Berry, J. Chem. Phys. 103, 1904 (1995),

[5] R.J. Hinde, R.S. Berry, and D.J. Wales, J. Chem. Phys. 96, 1376 (1992).

[6] C. Amitrano and R.S. Berry, Phys. Rev. Lett. 68, 729 (1992).

[7] H.A. Posch and W.G. Hoover, Phys. Rev. A 38, 473 (1988).

[8] H.A. Posch and W.G. Hoover, Phys. Rev. A 39, 2175 (1989).

[9] R.J. Hinde and R.S. Berry, J. Chem. Phys. 99, 2942 (1993).

[10] E. Yurtsever and N. Elmaci, Phys. Rev. A 55, 538 (1997).

[11] T.L. Beck, D.M. Leitner, and R.S. Berry, J. Chem. Phys. 89, 1681 (1988).

[12] D.J. Wales and R.S. Berry, J. Chem. Phys. 92, 4283 (1990).

[13] S.K. Nayak, R. Ramaswamy, and C. Chakravarty, Phys. Rev. E 51, 3376 (1995).

[14] V. Mehra and R. Ramaswamy, Phys. Rev. E 56, 2508 (1997).
[15] K-H. Kwon and B-Y. Park, J. Chem. Phys. 107, 5171 (1997).

[16] F. Calvo, J. Chem. Phys. 108, 6861 (1998).

[17] F. Calvo and P. Labastie, J. Phys. Chem. 102, 2051 (1998).

[18] E. Yurtsever, Phys. Rev. A 58, 377 (1998).

[19] E. Yurtsever, Europhys. Lett. 37, 91 (1997).

[20] J. Jellinek and D.H. Li, Phys. Rev. Lett. 62, 241 (1989).

[21] D.H. Li and J. Jellinek, Z. Phys. D: At., Mol. Clusters 12, 177 (1989).

[22] M.A. Miller and D.J. Wales, Mol. Phys. 89, 533 (1996).

[23] L.L. Lohr and C.H. Huben, J. Chem. Phys. 99, 6369 (1993).

[24] L.L. Lohr, Mol. Phys. 97, 977 (1999).

[25] A. Wolf, J.B. Swift, H.L. Swinney, and J.A. Vastano, Physica D 16, 285 (1985).

[26] M. Caglar, Ph.D. thesis, Princeton University, 1997; P.H. Baxendale, in Lyapunov Exponents, edited by L. Arnold and V. Wihstutz (Springer-Verlag, Berlin, 1984), p. 271. 